\title{
Hypoxic Preconditioning Improved Neuroprotective Effect of Bone Marrow-Mesenchymal Stem Cells Transplantation in Acute Glaucoma Models
}

\author{
Titiek Ernawati1,2, Gatut Suhendro ${ }^{3,4}$, I Ketut Sudiana5, Suhartono Taat Putra5, \\ Harjanto JM6, Sunarjo7, Agus Turchan 4,8, Fedik Abdul Rantam4,9,10,11 \\ ${ }^{1}$ Department of Ophthalmology, School of Medicine, Widya Mandala University, Surabaya, Indonesia \\ ${ }^{2} \mathrm{PHC}$ Hospital, Surabaya, Indonesia \\ ${ }^{3}$ Department of Ophthalmology, School of Medicine, Airlangga University, Surabaya, Indonesia \\ ${ }^{4}$ Dr. Soetomo General Hospital, Surabaya, Indonesia \\ ${ }^{5}$ Department of Pathology Anatomy, School of Medicine, Airlangga University, Surabaya, Indonesia \\ ${ }^{6}$ Department of Physiology, School of Medicine, Airlangga University, Surabaya, Indonesia \\ ${ }^{7}$ Department of Public Health, School of Medicine, Airlangga University, Surabaya, Indonesia \\ ${ }^{8}$ Department of NeuroSurgery, School of Medicine, Airlangga University, Surabaya, Indonesia \\ ${ }^{9}$ Laboratory of Stem Cell, Institute of Tropical Disease, Airlangga University, Surabaya, Indonesia \\ ${ }^{10}$ Regenerative Medicine \& Stem Cell Centre, Airlangga University, Surabaya, Indonesia \\ ${ }^{11}$ Laboratory of Virology and Immunology, Department of Microbiology, School of Veterinary Medicine, \\ Airlangga University, Surabaya, Indonesia \\ Email: titiek_ernawati@yahoo.com
}

Received 6 November 2015; accepted 27 March 2016; published 30 March 2016

Copyright (C) 2016 by authors and Scientific Research Publishing Inc.

This work is licensed under the Creative Commons Attribution International License (CC BY).

http://creativecommons.org/licenses/by/4.0/

c) (7) Open Access

\section{Abstract}

This study explored the novel strategy of hypoxic preconditioning of Bone Marrow Mesenchymal Stem Cells (BM-MSCs) before intra vitreal transplantation to improve neuroprotective effects of Retinal Ganglion Cells (RGCs) in Acute Glaucoma Models. The methods of this research were isolated mesenchymal stem cells from the bone marrow of adult wild-type Sprague-Dawley (SD) rats. BM-MSCs were cultured under normoxic or hypoxic (1\% oxygen for 24 hours) conditions. Normoxic or hypoxic BM-MSCs were transplanted intravitreally 1 week after ocular hypertension induction by acutely increasing IOP to $100-120 \mathrm{mmHg}$ for 60 minutes. Rats were killed 4 weeks after transplanted. Apoptosis was examined by tunnel assay and expression Brn3b (Brn3b = RGCs marker) by immunohistochemical analysis of the retina. Results showed that transplantation of hypoxic preconditioning BM-MSCs in acute glaucoma models resulted in a significant apoptosis decreasing $(p<0.05)$ and an significant increasing in RGCs $(p<0.05)$, as well as enhanced mor- 
phologic and functional benefits of stem cell therapy versus normoxic BM-MSCs transplantation. Conclusions: Hypoxic preconditioning enhances the capacity of BM-MSCs transplantation to improve neuroprotective effects of RGCs in Acute Glaucoma Models.

\title{
Keywords
}

\author{
Hypoxic Preconditioning, Transplantation, Bone Marrow-Mesenchymal Stem Cells, BM-MSCs, \\ Glaucoma, Neuroprotective
}

\section{Introduction}

Glaucoma represents a group of diseases defined by a characteristic optic neuropathy that is consistent with excavation and undermining of the neural and connective tissue elements of the optic disc and by the eventual development of distinctive patterns of visual dysfunction [1]. Glaucoma, a leading cause of irreversible visual loss, is characterized by loss of retinal ganglion cells (RGCs) and their axons over a period of many years. Glaucomatous optic neuropathy is characterized by changes in the optic disc and visual field defects [2] [3]. The pathophysiology of glaucoma is believed to be multifactorial [4] [5]. Elevated intraocular pressure (IOP) is one of the primary risk factors [1]. Until recently it was believed that elevated IOP plays a major role in RGCs apoptosis and it is also true that reduction of elevated IOP often helps in slowing down the progression of degenerative changes in glaucoma [4] [5].

The potential use of stem or progenitor cell transplantation to treat neurodegenerative diseases is a subject of intensive research [6]. Among stem cells that are candidates for transplantation in glaucoma patients, bone marrow mesenchymal stem cells (BMSCs) are preferred because they are available from autologous donation therefore eliminates ethical disputes and other concerns related to graft rejection [7]. Several in vitro and in vivo studies described conditions affecting on BM-MSCs to transdifferentiate into neural cells, either neurons or glial [8] [9]. BM-MSCs have a high predisposition to differentiate into preretinal-like cells with minimal time in culture. Brn3b and Ath5 gene expression increased markedly [10].

However, so far only a very small proportion of grafted cells have been shown to integrate into the host retina. Only $\sim 1 \%$ of intraocularly transplanted cells commonly migrate into the retina, whereas most remain as a bolus outside of the neural tissue [11]. Replacement of retinal ganglion cells (RGCs) presents an even greater challenge [11]. Neuroprotective strategies, in which transplanted cells protect endogenous neural tissue, have also shown promising results in animal models of retinal disease. The neuroprotective mechanism of action appears to include trophic factor secretion and/or modulation of inflammatory processes [11]. In addition, cell transplantation may activate endogenous repair mechanisms by modulating inhibitory signals to promote axonal regrowth and neuritic sprouting [12] [13].

Several strategies have been proposed to augment the longevity of engrafted cells in the hostile ischemic environment. These include the following: 1) treatment of the host tissue environment to make it more receptive for the donor cells; 2) in vitro manipulation (i.e., preconditioned donor cells); and 3) genetic modulation of the donor cells to make them more robust and resistant to apoptosis [14].

Hypoxic preconditioning BMSCs have been extensively investigated in various cell types, organs, animal models and humans [15]-[17]. Ischemic/hypoxic preconditioning activates endogenous defense mechanisms that show marked protective effects against multiple insults found in ischemic stroke and other acute attacks [15] [18]. Hypoxic preconditioning of transplanted BMSCs is an effective means of promoting their regenerative capability and therapeutic potential for the treatment of ischemic stroke [19].

Hypoxic preconditioning BMSCs increased expression of pro-survival and proangiogenic factors including hypoxia-inducible factor 1, angiopoietin-1, vascular endothelial growth factor and its receptor, Flk-1, erythropoietin, Bcl-2, and Bcl-xL. Cell death of hypoxic stem cells and caspase-3 activation in these cells were significantly lower compared with those in normoxic stem cells both in vitro and in vivo [17]. The hypoxia exposure up-regulated HIF-1 $\alpha$ and trophic/growth factors in BMSCs, including brain-derived neurotrophic factor (BDNF), glial cell-derived neurotrophic factor (GDNF), vascular endothelial growth factor (VEGF) and its receptor FIK-1, erythropoietin (EPO) and its receptor EPOR, stromal derived factor-1 (SDF-1) and its CXC chemokine 
receptor 4 (CXCR4) [19]. BMSCs have an inhibitory action on inflammatory responses, which exacerbate ischemic damage; and they express a variety of neurotrophic and growth factors that may show autocrine and paracrine effects after transplantation [7] [20].

Hypoxic preconditioning BMSCs with $1 \% \mathrm{O}_{2}$ was based on the state of each different organ levels of $\mathrm{O}_{2}$. Normal $\mathrm{O}_{2}$ levels inside the body vary by organ but are almost always substantially lower than the $20.8 \%$. Central nervous system (CNS) tissue $\mathrm{PO}_{2}$ values are conserved among mammalian species but vary by brain region. These range from as low as $0.55 \%(4.1 \mathrm{mmHg})$ in the midbrain to $8.0 \%(60 \mathrm{mmHg})$ in the pia. In rodents, the $\mathrm{PO}_{2}$ of cortical gray matter in rodents is $2.53 \%-5.33 \%\left(19-40 \mathrm{mmHg}\right.$ ) [21]. Normal $\mathrm{PO}_{2}$ at the mouse retinal surface was $21.7 \pm 0.8 \mathrm{mmHg}$ and the $\mathrm{PO}_{2}$ at the peak in the choroid was $42.0 \pm 1.2 \mathrm{mmHg}$. The minimum $\mathrm{PO}_{2}$ in the outer mouse retina was $4.2 \pm 0.5 \mathrm{mmHg}$, and in the inner mouse retina it was $5.0 \pm 0.5 \mathrm{mmHg}$ [22].

The culture in hypoxic preconditioning BMSCs $\left(1 \%-3 \% \mathrm{O}_{2}\right)$ may also be beneficial for the BMSCs, as this oxygen tension is more similar to the physiologic niche for MSC in the bone marrow $\left(2 \%-7 \% \mathrm{O}_{2}\right)$ [23]. Alteration of the duration of hypoxic conditions (oxygen concentrations $<2 \%$ ) has been shown to have a contrasting role in stem cells (SCs). In a short period of time (under $48 \mathrm{~h}$ ), hypoxia induced the proliferation, migration, and differentiation of various SCs, including BMSCs. Additionally, over a longer period of time ( $>72 \mathrm{~h}$ ), hypoxia can reduce SC proliferation and/or lead to programmed cell death [24]-[26].

In this study, we hypothesize that the hypoxic preconditioning approach in BMSCs transplantation therapy can lead to decrease apoptosis and increase RGCs in animal model of Acute Glaucoma.

\section{Material and Methods}

\subsection{Animal Experiment}

This experiment used adult (8 - 12 week old) male, weight 300 - 400 gram type Sprague-Dawley (SD) rats which was bred at Faculty of Veterinary Medicine, Airlangga University, Surabaya, Indonesia. All animals were housed in light- and temperature-controlled conditions and immunocompetent. All animal experiments operation prosedure were conducted with institutional of ethics approval in accordance with Animal Care and Use Committee (ACUC) the Faculty of Veterinary Medicine, Airlangga University, Surabaya, Indonesia. There were three groups of 24 SD rats.

\subsection{Isolation and Culture of Bone Marrow Mesenchymal Stem Cells (BM-MSCs)}

The procedure for isolation and culture of BM-MSCs were performed at Laboratory of Stem Cell, Institute of Tropical Disease, Airlangga University, Surabaya. Bone marrow-derived mesenchymal stem cells (BMSC) were cultured from the femoral and tibial bones of donor adult SD rats using a syringe and 20-gauge needle. The isolation method was performed using Ficoll 0.077 density (Invitrogen). After centrifugation by 1600 rpm, 30 min were isolated mononucleates cells from Buffy coat space and washed using growth medium serum free and followed centrifugation by $1600 \mathrm{rpm}$ in $10 \mathrm{~min}$. After that were cultured mononucleate cells in petridish $10 \mathrm{~cm}$ and growing in complete medium ( $\alpha$-MEM) (Invitrogen). Cultures were maintained at $37^{\circ} \mathrm{C}$ in a humidified atmosphere containing 5\% carbon dioxide. After 24 hours, nonadherent cells were discarded, and adherent cells were washed three times with phosphate-buffered saline solution (PBS). Fresh complete medium was added and replaced every 4 days. In second passage of mesenchymal stem cells phenotype were characterized by immunocytochemistry and flow cytometry. Mesenchymal stem cells were labeled used PKH26 (Sigma, USA).

\subsection{Phenotypic Characterization BM-MSCs}

\subsubsection{Immunocytochemistry}

Immunocytochemistry was used to characterize of phenotype rat Bone-Marrow derived Mesenchymal Stem Cell (rBMMSC) specific CD105 positive and CD45 negative. The cultured cells were plated onto coverslips and, after incubated at $37^{\circ} \mathrm{C}$ for $1-2$ hours, fixed with formaldehyde $10 \%$ for 15 minutes. The coverslips were then rinsed four times with PBS and let it dry for few minutes. The cells were blocked with for 1 hour in PBS containing 1\% bovine serum albumin (Sigma-Aldrich Corp) and 0.1\% Triton X-100 (Sigma-Aldrich Corp) for 45 min and then washed with PBS four times. FITC-labelled monoclonal antibody anti-rat CD105 and CD45 was applied to the cells and incubated for 60 minutes. Thereafter, the cells were rinsed with PBS twice and the cells 
were imaged using an Olympus inverted fluorescent microscope.

\subsubsection{Flow Cytometry}

Flow cytometry was used to characterize of phenotype rat Bone-Marrow derived Mesenchymal Stem Cell (rBMMSC) specific CD105 positive, CD90 positive, CD34 negative and CD45 negative. Cells were incubated for 15 min with erythrocyte lysis buffer at $41^{\circ} \mathrm{C}$ then were cells washed with PBS, and incubated with Dil-acLDL (acLDL) $(10 \mathrm{mg} / \mathrm{ml})$ for 1 hour at $37^{\circ} \mathrm{C}$. Cells were washed twice with plain medium, once with PBS, and fixed with $4 \%$ paraformaldehyde (PFA), and then cell were centrifuged at $1600 \mathrm{rpm}$ for $10 \mathrm{~min}$ and resuspended in PBS containing $0.2 \%$ FCS and 2 mM EDTA. Cells were incubated using Rat MSCs Analysis Kit (BD StemflowTM, BD Biosciences) with primary antibodies mouse anti-rat CD90, CD105, and negative antibodies containing CD45, CD34 for 40 minutes. Labeled cells were analyzed by FACSCalibur flow cytometer (BD Biosciences, Franklin Lakes, NJ, USA).

\subsection{Hypoxia Culture}

For hypoxic preconditioning (HP) treatment, cells were cultured until confluent. Fresh complete medium was added before hypoxia. Hypoxia treatment was controlled ProOx-C-chamber system (Biospherix, Redfield, NY) for 24 hours. The oxygen concentration in the chamber was maintained at $1 \%$ oksigen, with a residual gas mixture composed of $5 \%$ carbon dioxide and balanced nitrogen.

\subsection{Animal Model of Acute Glaucoma}

All the experiment manipulations were carried under anesthesia with ketamine $(50 \mathrm{mg} / \mathrm{kg})$ and xylazine (10 $\mathrm{mg} / \mathrm{kg}$ ) injected intramuscular. Local anesthetic $0.5 \%$ proparacaine hydrochloride was also applied topically to the cornea. The anterior chamber was cannuled with a 30-gauge needle connected to a syringe pump containing Balanced Salt Solution (BSS, Alcon Laboratories, Inc) under an operating microscope Inami. The IOP in experimental eyes was controlled with syringe pump, to maintain a pressure of 100 - $120 \mathrm{mmHg}$ for 60 minutes [27] [28]. The final IOP was achieved after $2-3$ minutes, and zero time for the ischemia was set. The flow of the pump was costantly monitored according to the pressure level. Retinal ischemia was achieved immediately with high IOPs, and it was confirmed by the blanching of the iris and retinal vascularitation. At the end of the $60 \mathrm{mi}-$ nutes of IOP elevation, the needle was removed, and reperfusion was confirmed by filling of the iris and retina vascularitation. Antibiotic drops Levofloxacin (LFX, Cendo Pharmaceuticals Inc. Indonesia) were applied topically after the prosedure. Experimental groups were divided into three groups of 8 rats each: 1) Glaucoma with transplant of normoxic BM-MSCs (N-BM-MSCs), 2) Glaucoma with transplant of hypoxic-precondisioning BM-MSCs (HP-BM-MSCs), 3) Glaucoma with no injection for precontrol (PC).

\subsection{Cell Preparation and Transplantation}

MSCs were labeled with PKH 26 to track and count the implanted cells. PKH 26 was added to culture medium for 2 hours before transplantation. Before transplantation, BM-MSCs in the HP group (HP-BM-MSCs) were treated for 24 hours with sublethal hypoxia (1\% oxygen) and then reoxygenated in 20\% oxygen for 2 hours. Seven days after IOP elevation rats $3 \times 10^{4}$ hypoxic preconditioning BM-MSCs (HP-BM-MSCs) in $3 \mu \mathrm{l}$ sterile PBS were transplanted into the vitreous cavity of the left eye under anesthesia with ketamine $(50 \mathrm{mg} / \mathrm{kg})$ and xylazine $(10 \mathrm{mg} / \mathrm{kg})$ injected intramuscular. Local anesthetic was also applied topically to the cornea. Cells were injected through the superior nasal retina using a 30 -gauge needle on a $5 \mu$ l glass Hamilton syringe. Care was taken to ensure that the lens was not damaged and that the retinal blood supply was not affected. In a separate group received normoxic BM-MSCs (N-BM-MSCs).

\subsection{Tissue Preparation}

Rats were carried under anesthesia with ketamine $(50 \mathrm{mg} / \mathrm{kg})$ and xylazine $(10 \mathrm{mg} / \mathrm{kg})$ injected intramuscular 4 weeks after IOP elevation. The eyes were enuclated and the globe was postfixed in $4 \%$ paraformaldehyde in 0.1 $\mathrm{mol} / \mathrm{L}$ phosphate-buffer saline for 4 hour. The cornea and lens were removed. Eyeballs were continuously sectioned along the vertical meridian. Cryosections, including a full length of retina passing through the optic nerve head and the superior and inferior regions of the eye, were chosen for comparison among groups. 


\subsection{Terminal Deoxynucleotidyl Transferase Biotin-dUPT Nick End Labeling (TUNEL) in Retina Sections}

A TUNEL staining kit (DeadEnd Fluorometric TUNEL system, Promega, Madison, Wis) was used to visualize apoptotic cells in retina sections. After 10 minutes of fixing by $10 \%$ buffered formalin phosphate (Fisher Scientific, Pittsburgh, Pa) and pretreatment with $-20^{\circ} \mathrm{C}$ ethanol/acetic acid (2:1) and $0.2 \%$ Triton X-100, the retina sections were incubated in an equilibration buffer as instructed by the kit. The TdT enzyme and nucleotide mix was then added at proportions specified by the kit for 75 minutes at room temperature. The slides were washed with 2X standard saline citrate washing buffer for 15 minutes and followed by three washings with PBS.

\subsection{Immunohistochemistry Analysis}

Immunohistochemistry on cryosections were blocked with goat serum for 1 hour at $37^{\circ} \mathrm{C}$ and then incubated with primary antibodies for 24 hours at $4^{\circ} \mathrm{C}$, followed by secondary antibodies for 1 hour at $37^{\circ} \mathrm{C}$. Primary antibodies included rat monoclonal antibody Brn3b. The positive cells were examined and mounted under inverted microscope.

\subsection{Statistic Analysis}

Experimental data were presented as mean \pm SD. Statistical calculations were performed using SPSS13.0 (SPSS Inc., USA). The indexes among groups were compared by multiple comparisons. Statistical significance was defined as $\mathrm{p}<0.05$. Data are expressed as the mean \pm standard error of mean.

\section{Result}

\subsection{Isolation and Culture of BM-MSCs}

The growth of the cells were observed for the first 24 hours, at which time the cells were mostly round or oval. After 24 hours, the majority of the cells were adherent to the plate and became spindle in shape. After 3 days, the cells grew to a confluence of $75 \%$ and ware arranged in swirling pattern [Figure 1(a)]. Before the cells were used for subsequent examination and transplantation, cell splitting procedure was performed 2 times. Before transplantation, BM-MSCs in the HP-BM-MSCs group were treated for 24 hours with sublethal hypoxia (1\% oxygen) [Figure 1(c)] and BM-MSCs in the N-BM-MSCs group which were still cultured in $20 \%$ oxygen [Figure 1(b)].

\subsection{The BM-MSCs Phenotypic Characterization}

\subsubsection{Immunocytochemistry}

The immunocytochemistry showed that the target cells expressed strong mesenchymal stem cell marker CD105 while weak expression of CD45 was noticed in N-BM-MSCs [Figure 2] and HP-BM-MSCs [Figure 3]. Expression of CD105 up to $80 \%$ on the surface membrane cells have important role, that mean were these cells already for transplantation, because it had showed an character of multipotent mesenchymal stem cells.

\subsubsection{Flow Cytometry}

The result of flowcytometry showed that majority of the target cells expressed strong CD90 and CD105, while CD45 and CD34 was expressed in small number of cells, which was noticed in N-BM-MSCs [Figure 4(a), Figure 4(b)] and HP-BM-MSCs [Figure 4(c), Figure 4(d)].

\subsection{PKH26 Analysis}

Labeling using PKH 26 (Sigma-Aldrich, USA) is performed before BM-MSCs being inserted into the eyeball, On examination using a microscope fluorescent would looked BM-MSCs that have been labeled with PKH 26 as in the yellowish orange [Figure 5].

On examination of the transplantation of BM-MSCs retinal tissue, it would looked fluorescence of the $\mathrm{PKH}$ 26 along the layers of the retina [Figure 6]. This is to prove the homing of exogenous BM-MSCs entered via intravitreal on retinal tissue. 


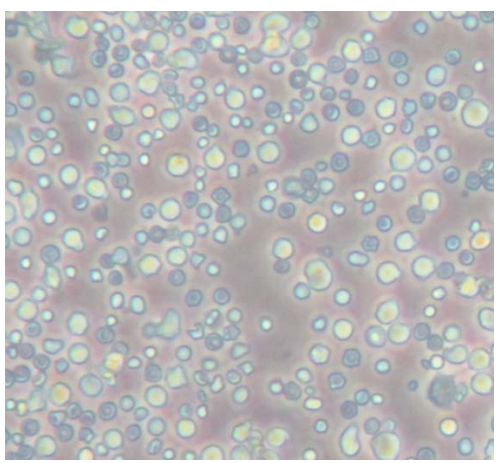

(a)

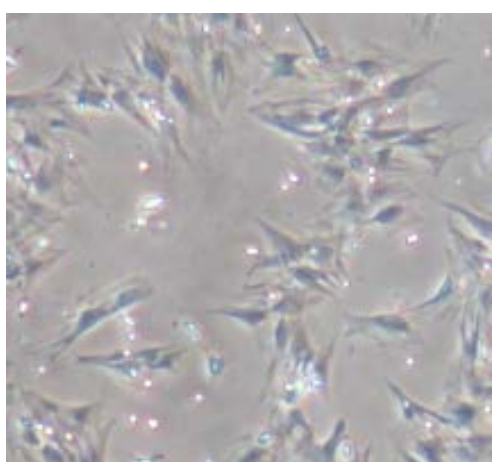

(b)

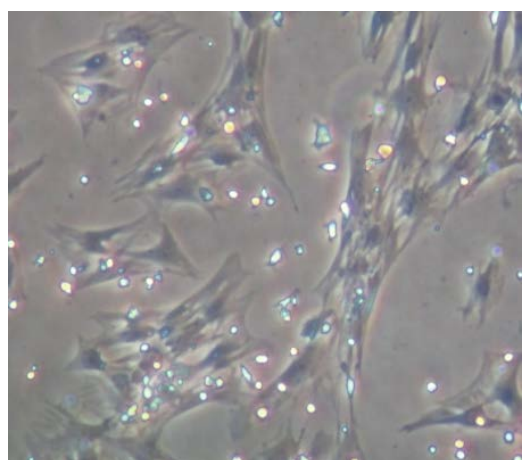

(c)

Figure 1. (a) Culture of BM-MSCs on the first 24 hours the cells were mostly round or oval in shape; (b) Culture of BMMSCs after 3 days the majority of the cells were adherent to the plate, showing spindle-shape with swirling-pattern colonies, and has reached confluence; (c) Culture of hypoxic precondisioning bone marrow mesenchymal stem cells (HP-BM-MSCs) showing spindle-shape with swirling-pattern colonies, and has reached conluence same with N-BM-MSCs (inverted microscope, 200× magnification).

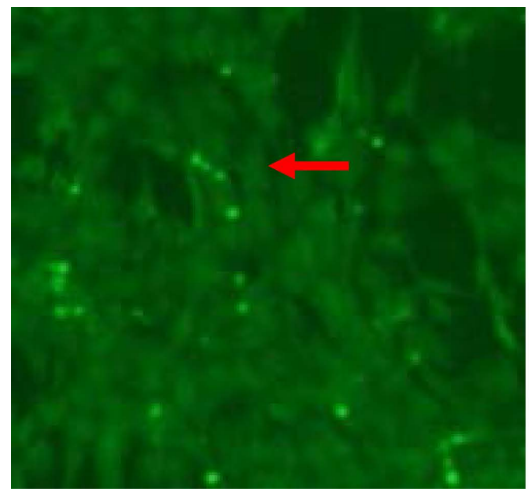

(a)

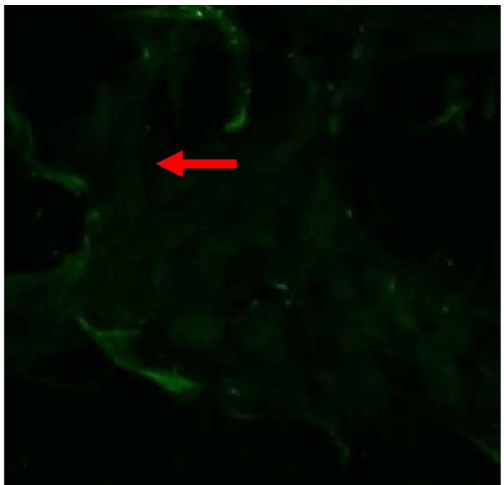

(b)

Figure 2. Characterization of N-BM-MSCs by immunocytochemistry. Expression of (a) CD105, (b) CD45. Expression of CD105 up to $80 \%$ (inverted microscope, 200× magnification).

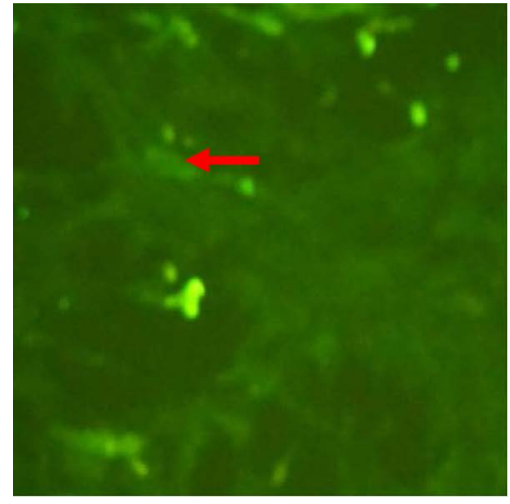

(a)

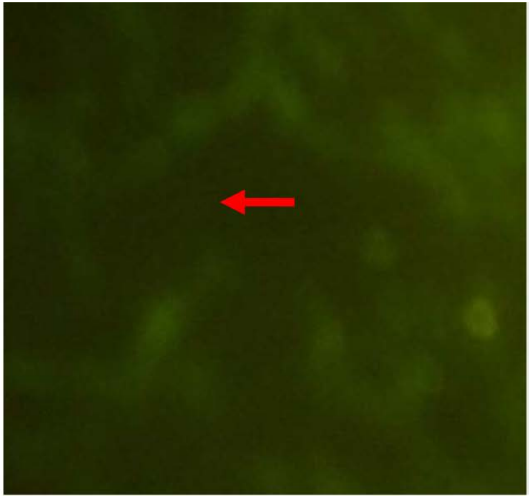

(b)

Figure 3. Characterization of HP-BM-MSCs by immunocytochemistry. Expression of (a) CD105, (b) CD45 Expression of CD105 up to $80 \%$ (inverted microscope, 200× magnification).

\subsection{TUNEL Analysis}

Examination of apoptosis in RGCs layer with Tunnel Assay painting to determine the number of cells undergoing apoptosis in retinal ganglion cell layer. The result of Tunnel Assay examination would show the lowest 


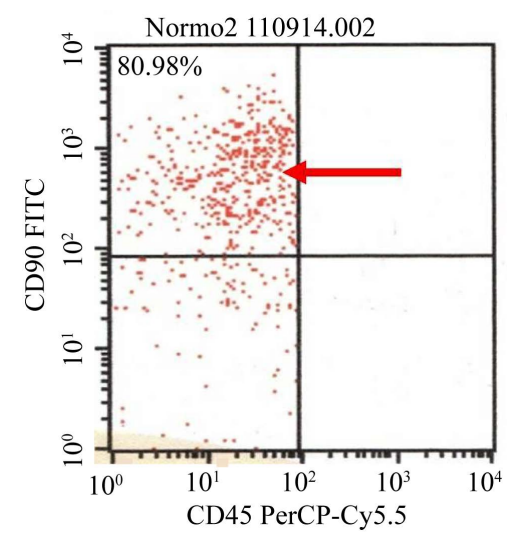

(a)

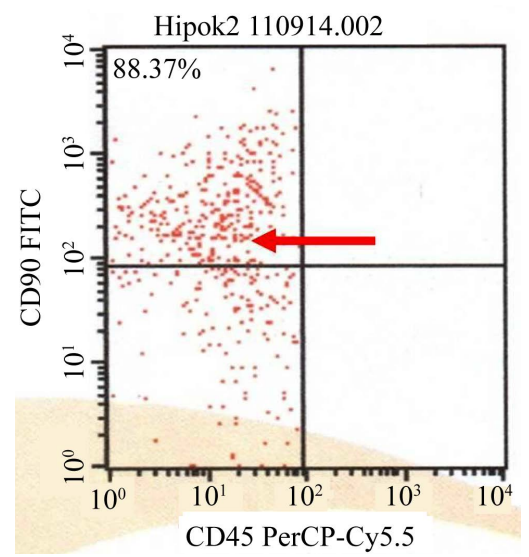

(c)

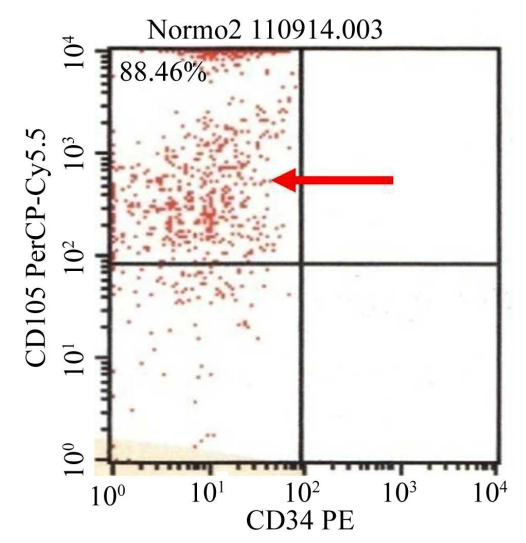

(b)

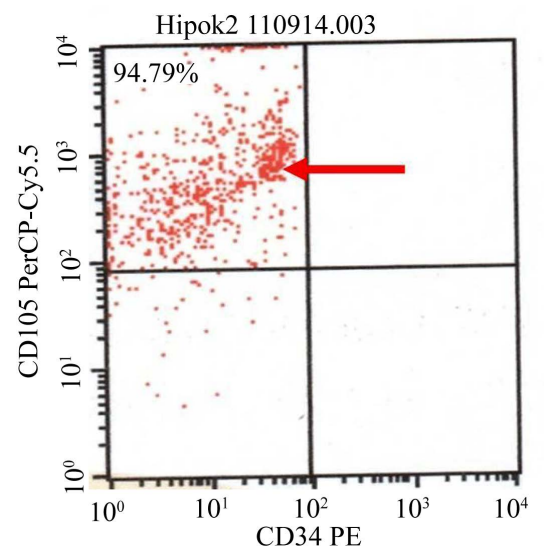

(d)

Figure 4. (a) (b) Characterization of N-BM-MSCs by flowcytometry CD90 positive (80.98\%), 105 positive (88.46\%), CD45 negative and CD34 negative; (c) (d) Characterization of HP-BM-MSCs by flowcytometry CD90 positive (88.37\%), 105 positive (94.79\%), CD45 negative and CD34 negative.

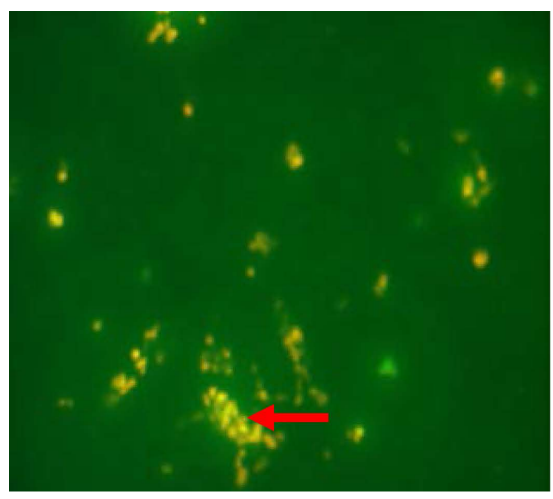

(a)

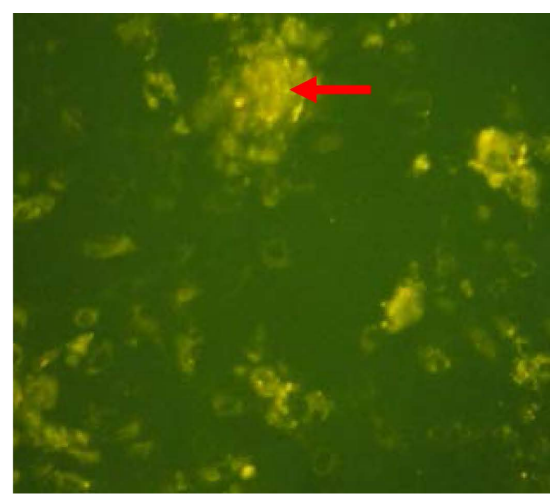

(b)

Figure 5. PKH26 analysis on BM-MSCs. PKH26 fluoresces in the yellow-orange region of the spectrum. (a) N-BM-MSCs group in second passage; (b) HP-BM-MSCs group in second passage. Arrow showed PKH26 fluoresces in the yellow-orange region on BM-MSCs (inverted microscope, 200× magnification).

number of undergoing apoptosis cells in the HP-BM-MSCs group compared with the other groups [Figure 7]. Apoptotic cells means on HP-BM-MSCs group $0.017 \pm 0.041$ would show the lowest number [Figure 8]. 


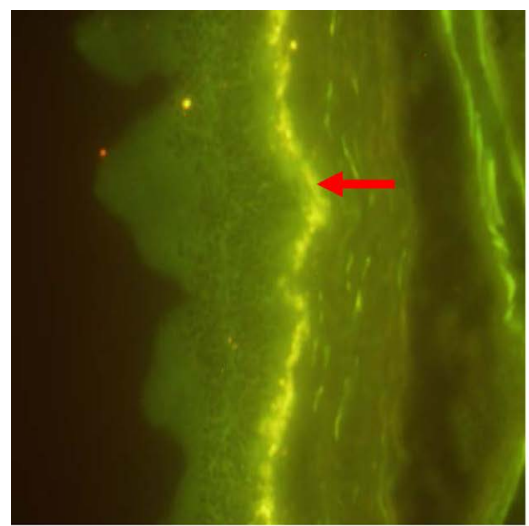

(a)

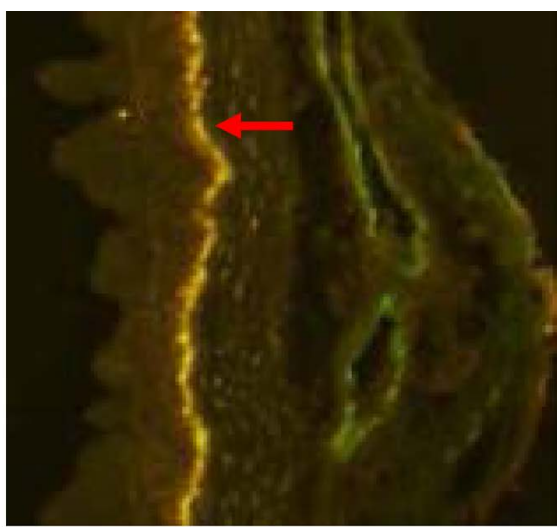

(b)

Figure 6. PKH26 analysis on retinal ganglion cells layer four week after transplanted rom acute glaucoma animal models. (a) N-BM-MSCs group four week after transplanted N-BM-MSCs via intravitreal from acute glaucoma animal models. Arrow showed homing N-BM-MSCs in retinal ganglion cells layer with PKH26 fluoresces in the yellow-orange region; (b) HP-BM-MSCs group four week after transplanted PH-BM-MSCs via intravitreal from acute glaucoma animal models. Arrow showed homing PH-BM-MSCs in retinal ganglion cells layer (inverted microscope, 200× magnification).

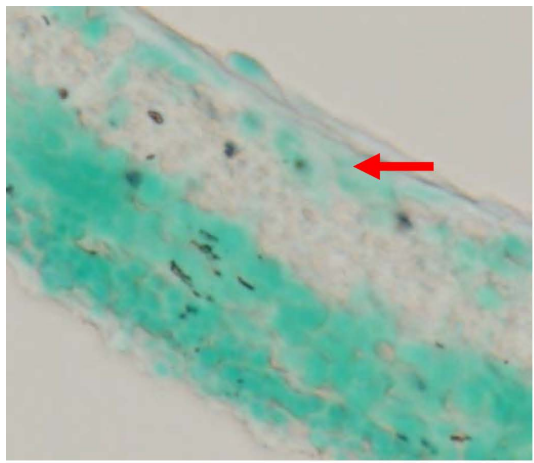

(a)

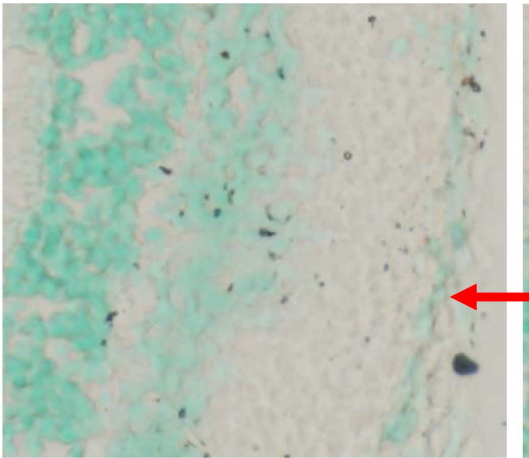

(b)

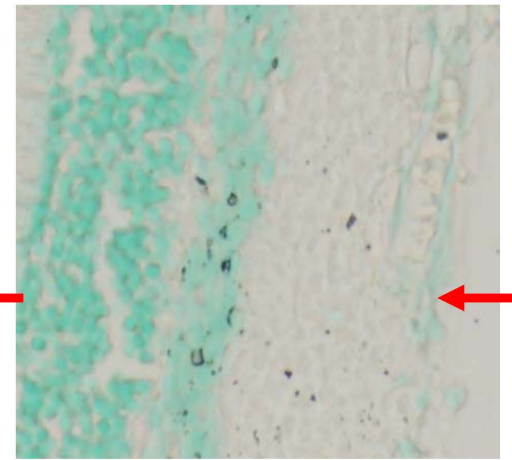

(c)

Figure 7. TUNEL Analysis on RGCs layer. (a) Control group; (b) N-BM-MSCs group; (c) HP-BM-MSCs group. Arrow showed apoptotic cells in retinal ganglion cells layer (inverted microscope, 200× magnification).

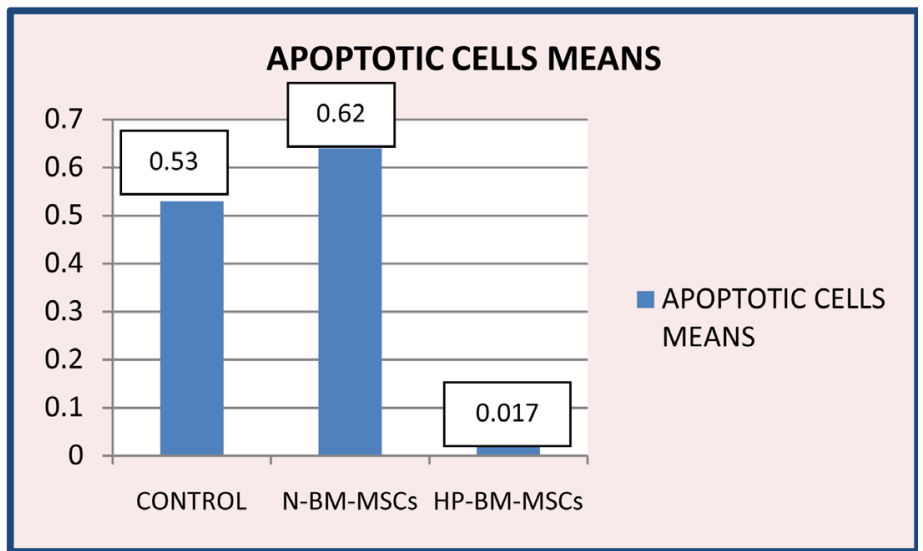

Figure 8. Diagram of TUNEL Analysis on control group, N-BM-MSCs group and HP-BM-MSCs group. Apoptotic cells means on control group $0.533 \pm 0.137$; N-BM-MSCs group $0.623 \pm 0.278$; HP-BM-MSCs group $0.017 \pm 0.041$.

Statistical data apoptotic cells mean the normality in each group was tested by using One Sample Kolmogorov-Smirnov showing result of a normal distribution. Then continued Multiple Comparisons Test to determine 
the differences between groups with $\mathrm{p}=0.05$. The results found a significant reduction in the number of cells undergoing apoptosis with $\mathrm{p}<0.05$ in the HP-BM-MSCs group against control group and N-BM-MSCs group.

\subsection{Immunohistochemistry}

Examination of Brn3b expression in retinal tissue with immunohistochemical staining is to determine the number of RGCs [Figure 9]. The result of Brn3b expression immunohistochemistry examination showed an increase in the number of RGCs in HP-BM-MSCs group (40.950) compared with other groups [Figure 10]. The lowest was in the control group.

Statistical data Brn3b means (RGCs) means the normality of each group was tested by using One Sample Kolmogorov-Smirnov showing results of a normal distribution. Then continued with Multiple Comparisons Test to determine the differences between groups with $\mathrm{p}=0.05$. The results found a significant enhancement in the number of RGCs with $\mathrm{p}<0.05$ in the HP-BM-MSCs group against control and N-BM-MSCs group.

\section{Discussion}

MSC is multipotent stem cell, that has a plasticity properties, and also have many kinds of protein as markers or signal expressed on the surface membrane cell have important role in communication between cell. CD105 is relative stable markers of MSC, because many kinds of markers can be used for detection of MSCs like CD90,

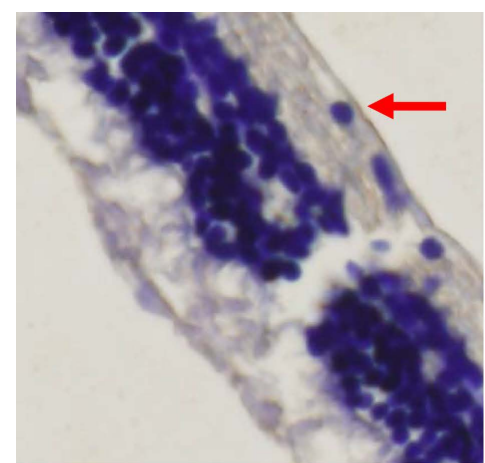

(a)

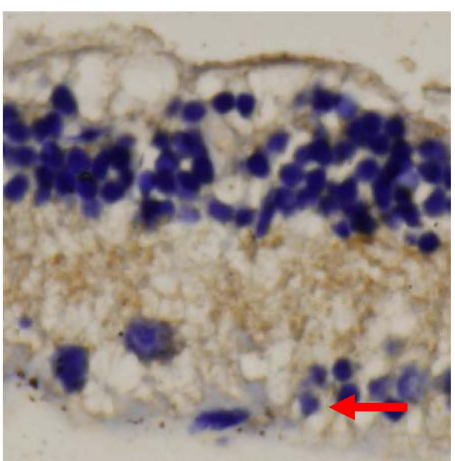

(b)

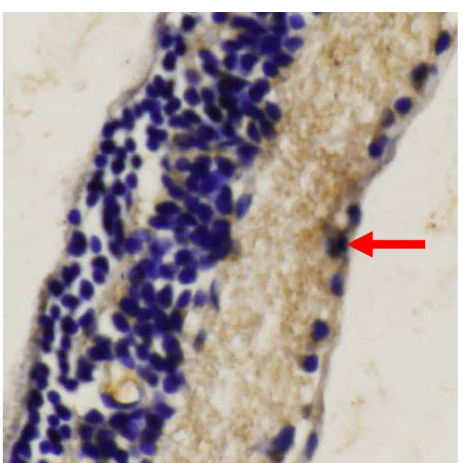

(c)

Figure 9. Immunohistochemistry analysis of Brn3b expression four week after transplanted from acute glaucoma animal models. (a) Control group five weeks after intra ocular pressure reduction on acute glaucoma animal models. Arrow showed Brn3b expression on retinal ganglion cells layer; (b) N-BM-MSCs group four week after transplanted N-BM-MSCs. Arrow showed Brn3b expression on retinal ganglion cells layer; (c) HP-BM-MSCs group four week after transplanted PH-BM-MSCs. Arrow showed Brn3b expression on retinal ganglion cells layer (inverted microscope, $400 \times$ magnification).

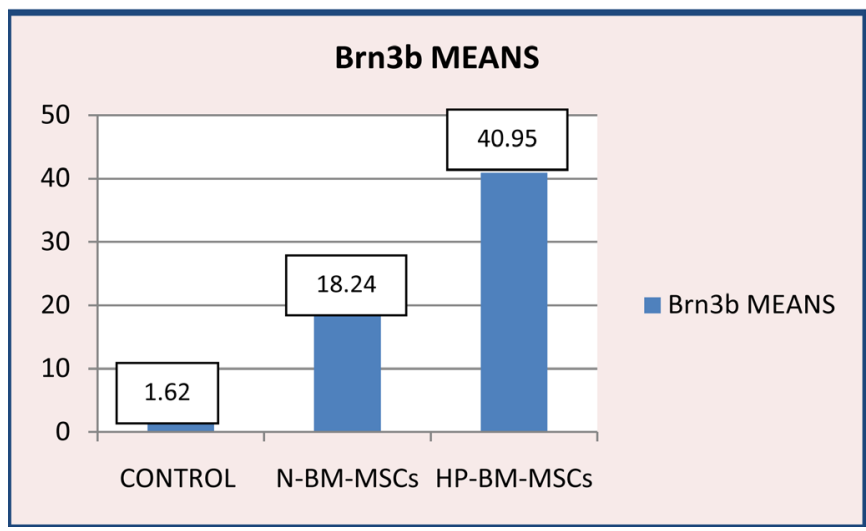

Figure 10. Diagram of Brn3b expression on control group, N-BM-MSCs group and HP-BM-MSCs group. Brn3b expression means on control group $1.167 \pm 0.592$; N-BM-MSCs group $18.245 \pm 4.910$; HP-BM-MSCs group $40.950 \pm 9.431$. 
CD73 and cells did not express CD34, CD11b, CD19, CD45 and HLA-DR [29]-[31]. In this research, we used HP-BM-MSCs with expression of CD90 positive (88.37\%), 105 positive (94.79\%) and N-BM-MSCs with expression of CD90 positive (80.98\%), 105 positive (88.46\%).

BM-MSCs have the ability to differentiate into neuroectodermal cell types in vitro and also in vivo after transplantation into the human brain and spinal cord [9].

BM-MSCs were cultivated in a neuroinduction mix medium for $24 \mathrm{~h}$ showed that Brn3b and Ath5 gene expression increased markedly. These results suggest that BM-MSCs have a high predisposition to differentiate into preretinal-like cells with minimal time in culture [10].

BM-MSCs have an important role in tissue engineering and regenerative medicine due to their immunogenicity, for the ability to perform immunomodulatory and anti-inflammatory functions. They have ability to secrete multiple bioactive molecules able of stimulating recovery of injured cells [32].

Intraocular transplantation of stem cells for retinal therapy can be achieved via two approaches, either subretinally or intravitreally, with each technique possessing advantages and disadvantages for particular applications. Subretinal injections leave cells physically constrained adjacent to the outer retina and near to rich blood supply, whereas intravitreal injections are technically simpler and provide direct access to the inner retina [31]. In this research, we used intravitreally transplanted cells.

Glaucoma is a common neurodegenerative disease of the inner retina. In the context of inner retinal disease, intravitreal injections are likely to be more applicable than subretinal injections. the ECM of the inner basal lamina is neither necessary nor sufficient to prevent migration of transplanted cells into the neural retina. In contrast, glial reactivity was associated with poor graft migration. Targeted disruption of glial reactivity dramatically improved the structural integration of intravitreally transplanted cells [31].

BMSCs subretinal transplantation could inhibit photoreceptor apoptosis and slow down retinal damage in light-damaged rat eyes. Transplanted BMSCs survived in the subretinal space and ONL but did not differentiate into neural cells or retinal cells. BMSCs have the capacity to produce some kinds of trophic factors in the lightdamaged model eye and in vitro cultivation with light-damaged SHR, which might have trophic and protective effects on light-damaged retina [33].

In ischemic/hypoxic environment, BMSC transplantation has been low homing and survival rates of BMSCs and of the observation that the functional benefits after BMSC transplantation has been attributed to increased trophic support but not cell replacement cortex cerebri is extremely low (e.g. $~ 0.01 \%$ of total inj [34]. The homing of systemically administered BMSCs to the ischemic ected cells) [35]. Johnson, 2010 and others have reported that only $\sim 1 \%$ of intraocularly transplanted cells commonly migrate into the retina, whereas most remain as a bolus outside of the neural tissue.

Strategies have been proposed to augment the longevity of engrafted cells in the hostile ischemic environment is in vitro manipulation (i.e., preconditioned donor cells) [14]. In this research, we used hypoxic preconditioning $1 \% \mathrm{O}_{2}$.

HP treatment promotes survival of transplanted cells in the ischemic brain and heart [15] [17] [36]. Hypoxic preconditioning increased expression of several trophic/growth factors in MSCs [19].

In ischemic/hypoxic preconditioning and neuroprotection against ischemic injury, HIF- $1 \alpha$ and HIF- $1 \beta$ play central roles [36] [37] [39]. Its translocation and activation in the nucleus result in production of several downstream genes such as vascular endothelial growth factor (VEGF), erythropoietin (EPO), glial cell line-derived neurotrophic factor (GDNF), brain-derived neurotrophic factor (BDNF), nerve growth factor (NGF) all are important for brain protection and tissue regeneration [36]-[38].

Transplantation of hypoxic versus normoxic BM-MSCs after myocardial infarction resulted in an increase in angiogenesis, as well as enhanced morphologic and functional benefits of stem cell therapy. Hypoxic preconditioning increased expression of pro-survival and proangiogenic factors including hypoxia-inducible factor 1, angiopoietin-1, vascular endothelial growth factor and its receptor, Flk-1, erythropoietin, Bcl-2, and Bcl-xL. Cell death of hypoxic stem cells and caspase-3 activation in these cells were significantly lower compared with that in normoxic stem cells both in vitro and in vivo [17].

HP can decrease apoptosis of neurons through induction of hypoxia-inducible factor- $1 \alpha$ (HIF-1 $\alpha$ ) [39] and protect myocytes from hypoxia and reperfusion injury [18].

Microglia, cells of the monocyte/macrophage lineage in the brain, have been implicated in the pathogenesis of a number of neurodegenerative [40]. As part of the innate immune defense mechanism, microglia can defend the central nervous system against damage, but increasing evidence suggest that excessive or sustained microglia 
activation can significantly contribute to acute and chronic neuropathologies and apoptotic cell death. Dysregulation of microglial cytokine production could thereby promote harmful actions of the defense mechanisms, result in direct neurotoxicity, as well as disturb neural cell functions [41]-[43].

\section{Conclusions}

Hypoxic preconditioning of transplanted cells provides a new and effective strategy for cell-based therapy in the treatment of ischemic diseases. Hypoxic preconditioning enhances the capacity of BM-MSCs to improve neuroprotective effects in Acute Glaucoma Models.

The mechanism of the therapeutic benefits must be multifaceted, and at least involve enhanced expression and release of trophic/growth factors that provide autocrine and paracrine effects of protection and stimulation of regenerative responses.

\section{Acknowledgements}

We thank to all researcher team at the Stem Cell Center, Institute of Tropical Disease Airlangga University Surabaya, East Java, Indonesia.

\section{References}

[1] Skuta, G.L., Cantor, L.B. and Weiss, J.S. (2009) Basic and Clinical Science Course Glaucoma. Vol. 2009-2010, Section 10, San Francisco.

[2] Gupta, N. and Weinreb, R.N. (1997) New Definitions of Glaucoma. Current Opinion in Ophthalmology, 8, 38-41. http://dx.doi.org/10.1097/00055735-199704000-00007

[3] Quigley, H.A. (1999) Neuronal Death in Glaucoma. Progress in Retinal and Eye Research, 18, 39-57. http://dx.doi.org/10.1016/S1350-9462(98)00014-7

[4] Klein, B.E., Klein, R., Sponsel, W.E., Franke, T., Cantor, L.B., Martone, J., et al. (1992) Prevalence of Glaucoma: The Beaver Dam Eye Study. Ophthalmology, 99, 1499-1504. http://dx.doi.org/10.1016/S0161-6420(92)31774-9

[5] Tielsch, J.M., Katz, J., Singh, K., Quigley, H.A., Gottsch, J.D., Javitt, J., et al. (1991) A Population-Based Evaluation of Glaucoma Screening: The Baltimore Eye Survey. American Journal of Epidemiology, 134, 1102-1110.

[6] Johnson, T.V., Bull, N.D. and Martin, K.R. (2010) Identification of Barriers to Retinal Engraftment of Transplanted Stem Cells. Investigative Ophthalmology \& Visual Science, 51, 960-970. http://dx.doi.org/10.1167/iovs.09-3884

[7] Malgieri, A., et al. (2010) Bone Marrow and Umbilical Cord Blood Human Mesenchymal Stem Cells: State of the Art. International Journal of Clinical and Experimental Medicine, 3, 248-269.

[8] Otify, D.Y., Youssef, E.A., Nagy, N.B., Marei, M.K. and Youssif, M.I. (2014) Transdifferentiation of Bone Marrow Mesenchymal Stem Cells into Neural Cells via Cerebrospinal Fluid. Biomedicine and Biotechnology, 2, 66-79.

[9] Hermann, A., Liebau, S., Gastl, R., Fickert, S., Habisch, H.J., Fiedler, J., Schwarz, J., Brenner, R. and Storch, A. (2006) Comparative Analysis of Neuroectodermal Differentiation Capacity of Human Bone Marrow Stromal Cells Using Various Conversion Protocols. Journal of Neuroscience Research, 83, 1502-1514. http://dx.doi.org/10.1002/jnr.20840

[10] Garza, M.T.G. and Cuevas, J.E.M. (2012) Rat Adult Stem Cell Differentiation into Immature Retinal Cells. Stem Cell Discovery, 2, 62-69. http://dx.doi.org/10.4236/scd.2012.22010

[11] Bull, N.D., Johnson, T.V. and Martin, K.R. (2008) Stem Cells for Neuroprotection in Glaucoma. Progress in Brain Research, 173, 511-519. http://dx.doi.org/10.1016/S0079-6123(08)01135-7

[12] Charalambous, P., Hurst, L.A. and Thanos, S. (2008) Engrafted Chicken Neural Tube-Derived Stem Cells Support the Innate Propensity for Axonal Regeneration within the Rat Optic Nerve. Investigative Ophthalmology \& Visual Science, 49, 3513-3524. http://dx.doi.org/10.1167/iovs.07-1473

[13] Zhang, Y., Klassen, H.J., Tucker, B.A., Perez, M.T. and Young, M.J. (2007) CNS Progenitor Cells Promote a Permissive Environment for Neurite Outgrowth via a Matrix Metalloproteinase-2-Dependent Mechanism. Journal of Neuroscience, 27, 4499-4506. http://dx.doi.org/10.1523/JNEUROSCI.0200-07.2007

[14] Wang, X. (2009) Hsp20-Engineered Mesenchymal Stem Cells Are Resistant to Oxidative Stress via Enhanced Activation of Akt and Increased Secretion of Growth Factors. Wiley Online Library.

[15] Theus, M.H. (2009) In Vitro Hypoxic Preconditioning of Embryonic Stem Cells as a Strategy of Promoting Cell Survival and Functional Benefits after Transplantation into the Ischemic Rat Brain. Experimental Neurology, 120, 656670.

[16] Hausenloy, D.J. and Yellon, D.M. (2009) Preconditioning and Postconditioning: Underlying Mechanisms and Clinical 
Application. Atherosclerosis, 204, 334-341. http://dx.doi.org/10.1016/j.atherosclerosis.2008.10.029

[17] Hu, X., Yu, S.P., Fraser, J.L., Lu, Z., Ogle, M.E., Wang, J.A. and Wei, L. (2008) Transplantation of Hypoxia-Preconditioned Mesenchymal Stem Cells Improves Infarcted Heart Function via Enhanced Survival of Implanted Cells and Angiogenesis. The Journal of Thoracic and Cardiovascular Surgery, 135, 799-808. http://dx.doi.org/10.1016/j.jtcvs.2007.07.071

[18] Yu, S.P., Wei, Z. and Wei, L. (2013) Preconditioning Strategy in Stem Cell Transplantation Therapy. Translational Stroke Research, 4, 76-88. http://dx.doi.org/10.1007/s12975-012-0251-0

[19] Wei, L., Fraser, J.L., Yang, L.Z., Hu, X. and Yu, S.P. (2012) Transplantation of Hypoxia Preconditioned Bone Marrow Mesenchymal Stem Cells Enhances Angiogenesis and Neurogenesis after Cerebral Ischemia in Rats. Neurobiology of Disease, 46, 635-645. http://dx.doi.org/10.1016/j.nbd.2012.03.002

[20] Tse, W.T., Pendleton, J.D., Beyer, W.M., Egalka, M.C. and Guinan, E.C. (2003) Suppression of Allogeneic T-Cell Proliferation by Human Marrow Stromal Cells: Implications in Transplantation. Transplantation, 75, 389-397. http://dx.doi.org/10.1097/01.TP.0000045055.63901.A9

[21] Erecinska, M. and Silver, I.A. (2001) Tissue Oxygen Tension and Brain Sensitivity to Hypoxia. Respiration Physiology, 128, 263-276. http://dx.doi.org/10.1016/S0034-5687(01)00306-1

[22] Yu, D.-Y. and Cringle, S.J. (2006) Oxygen Distribution in the Mouse Retina. Investigative Ophthalmology \& Visual Science, 47, 1109-1112. http://dx.doi.org/10.1167/iovs.05-1118

[23] Grayson, W.L., Zhao, F., Izadpanah, R., Bunnell, B. and Ma, T. (2006) Effects of Hypoxia on Human Mesenchymal Stem Cell Expansion and Plasticity in 3D Constructs. Journal of Cellular Physiology, 207, 331-339. http://dx.doi.org/10.1002/jcp.20571

[24] Lee, S.H., Lee, Y.J. and Han, H.J. (2011) Role of Hypoxia-Induced Fibronectin-Integrin $\beta 1$ Expression in Embryonic Stem Cell Proliferation and Migration: Involvement of PI3K/Akt and FAK. Journal of Cellular Physiology, 226, 484-493. http://dx.doi.org/10.1002/jcp.22358

[25] Prado-Lopez, S., Conesa, A., Armiñán, A., Martinez-Losa, M., Escobedo-Lucea, C., Gandia, C., et al. (2010) Hypoxia Promotes Efficient Differentiation of Human Embryonic Stem Cells to Functional Endothelium. Stem Cells, 28, 407-418.

[26] Lee, S.H., Suh, H.N., Lee, Y.J., Seo, B.N., Ha, J.W. and Han, H.J. (2012) Midkine Prevented Hypoxic Injury of Mouse Embryonic Stem Cells through Activation of Akt and HIF-1 $\alpha$ via Low-Density Lipoprotein Receptor-Related Protein-1. Journal of Cellular Physiology, 227, 1731-1739. http://dx.doi.org/10.1002/jcp.22897

[27] Adachi, M., Takahashi, K., Nishikawa, M., Miki, H. and Uyama, M. (1996) High Intraocular Pressure-Induced Ischemia and Reperfusion Injury in the Optic Nerve and Retina in Rat. Graeff's Archieve for Clinical and Experimental Ophthalmology, 234, 445-451. http://dx.doi.org/10.1007/BF02539411

[28] Windisch, B.K., LeVatte, T.L., Archibald, M.L. and Chauhan, B.C. (2009) Induction of Heat Shock Proteins 27 and 72 in Retinal Ganglion Cells after Acute Pressure-Induced Ischaemia. Clinical \& Experimental Ophthalmology, 37, 299-307.

[29] Slater, K., Katie, P., Thomson, J., et al. (2013) Synthetic Peptide Coated Surface for Culture of Human Mesenchymal Stem Cell in a Defined and Xeno-Free Medium. Adult Stem Cell Therapy and Regenerative Medicine, Cleveland Marriott Downtown at Key Center, Cleveland, 19-21 August 2013, 53.

[30] Xu, W. and Xu, G.X. (2011) Mesenchymal Stem Cells for Retinal Diseases. International Journal of Ophthalmology, 4, 413-421.

[31] Johnson, T.V., Bull, N.D. and Martin, K.R. (2010) Identification of Barriers to Retinal Engraftment of Transplanted Stem Cells. Investigative Ophthalmology \& Visual Science, 51, 960-970. http://dx.doi.org/10.1167/iovs.09-3884

[32] Taran, R., Mamidi, M.K., Singh, G., Dutta, S., Parhar, I.S., John, J.P., Bhonde, R., Pal, R. and Das, A.K. (2014) In Vitro and in Vivo Neurogenic Potential of Mesenchymal Stem Cells Isolated from Different Sources. Journal of Biosciences, 39, 157-169. http://dx.doi.org/10.1007/s12038-013-9409-5

[33] Zhang, Y. and Wang, W. (2010) Effects of Bone Marrow Mesenchymal Stem Cell Transplantation on Light-Damaged Retina. Investigative Ophthalmology \& Visual Science, 51, 3742-3748. http://dx.doi.org/10.1167/iovs.08-3314

[34] Hess, D.C. and Borlongan, C.V. (2008) Stem Cells and Neurological Diseases. Cell Proliferation, 41, 94-114. http://dx.doi.org/10.1111/j.1365-2184.2008.00486.X

[35] Chen, J., Li, Y., Wang, L., Zhang, Z., Lu, D., Lu, M. and Chopp, M. (2001) Therapeutic Benefit of Intravenous Administration of Bone Marrow Stromal Cells after Cerebral Ischemia in Rats. Stroke, 32, 1005-1011. http://dx.doi.org/10.1161/01.STR.32.4.1005

[36] Ogle, M.E., Yu, S.P. and Wei, L. (2009) Primed for Lethal Battle: A Step Forward to Enhance the Efficacy and Efficiency of Stem Cell Transplantation Therapy. The Journal of Thoracic and Cardiovascular Surgery, 138, 527. 
http://dx.doi.org/10.1016/j.jtcvs.2009.06.003

[37] Semenza, G.L. (2011) Hypoxia-Inducible Factor 1: Regulator of Mitochondrial Metabolism and Mediator of Ischemic Preconditioning. Biochimica et Biophysica Acta-Molecular Cell Research, 1813, 1263-1268. http://dx.doi.org/10.1016/j.bbamcr.2010.08.006

[38] Chen, X., Li, Y., Wang, L., Katakowski, M., Zhang, L., Chen, J., et al. (2002) Ischemic Rat Brain Extracts Induce Human Marrow Stromal Cell Growth Factor Production. Neuropathology, 22, 275-279. http://dx.doi.org/10.1046/j.1440-1789.2002.00450.x

[39] Liu, J., Narasimhan, P., Yu, F. and Chan, P.H. (2005) Neuroprotection by Hypoxic Preconditioning Involves Oxidative Stress-Mediated Expression of Hypoxiainducible Factor and Erythropoietin. Stroke, 36, 1264-1269. http://dx.doi.org/10.1161/01.STR.0000166180.91042.02

[40] Danton, G.H. and Dietrich, W.D. (2003) Inflammatory Mechanisms after Ischemia and Stroke. Journal of Neuropathology \& Experimental Neurology, 62, 127-136. http://dx.doi.org/10.1093/jnen/62.2.127

[41] Hanisch, U.K. (2002) Microglia as a Source and Target of Cytokines. Glia, 40, 140-155. http://dx.doi.org/10.1002/glia.10161

[42] Ohmi, K., Greenberg, D.S., Rajavel, K.S., Ryazantsev, S., Li, H.H. and Neufeld, E.F. (2003) Activated Microglia in Cortex of Mouse Models of Mucopolysaccharidoses I and IIIB. Proceedings of the National Academy of Sciences of the United States of America, 100, 1902-1907. http://dx.doi.org/10.1073/pnas.252784899

[43] Uchiyama, T., Engelman, R.M., Maulik, N. and Das, D.K. (2004) Role of Akt Signaling in Mitochondrial Survival Pathway Triggered by Hypoxic Preconditioning. Circulation, 109, 3042-3049.

http://dx.doi.org/10.1161/01.CIR.0000130647.29030.90 